\title{
JACQUES MÉLÈSE
}

\section{Quelle est la place de la recherche opérationnelle dans les programmes de gestion à court terme ?}

Revue française d'automatique, d'informatique et de recherche opérationnelle. Recherche opérationnelle, tome 2, no V3 (1968), p. 3-8.

<http://www.numdam.org/item?id=RO_1968_2_3_3_0>

(C) AFCET, 1968, tous droits réservés.

L'accès aux archives de la revue « Revue française d'automatique, d'informatique et de recherche opérationnelle. Recherche opérationnelle » implique l'accord avec les conditions générales d'utilisation (http://www.numdam.org/ legal.php). Toute utilisation commerciale ou impression systématique est constitutive d'une infraction pénale. Toute copie ou impression de ce fichier doit contenir la présente mention de copyright.

\section{Numdam}

Article numérisé dans le cadre du programme

Numérisation de documents anciens mathématiques

http://www.numdam.org/ 
R.I.R.O.

(2e année, No 14,1968 , p. 3-8)

\title{
QUELLE EST LA PLAGE DE LA REGHERGHE OPÉRATIONNELLE DANS LES PROGRAMMES DE GESTION A GOURT TERME?
}

\author{
par Jacques MéLìse \\ Président de l'AUROC \\ Directeur des Études de la CEGOS
}

Résumé. - Dans cet article, l'auteur constate que, bien que les modèles théoriques soient nombreux, les études et les mises en applications de programmes de gestion à court terme échappent souvent aux chercheurs opérationnels et sont traités principalement par des informaticiens et des organisateurs. Il recherche les raisons de cet état de fait, ce qui le conduit à poser un certain nombre de questions sur l'adéquation du style et des techniques classiques de la Recherche Opérationnelle aux problèmes de programmation à court terme.

Le titre de cet article est une interrogation et il sera suivi de nombreuses autres questions. Pourquoi ? Parce que dans le domaine des programmes à court terme on ne peut manquer d'être frappé par la disparité entre les études théoriques et les applications : les revues spécialisées regorgent de modèles et d'algorithmes concernant l'ordonnancement, l'affectation, les transports, les problèmes combinatoires en général, alors que, dans tous les pays, les réalisations pratiques de Recherche Opérationnelle sur ces sujets sont peu nombreuses.

Bien sûr, on va m'objecter que les applications du P.E.R.T. courent les rues; certes, mais c'est si peu de la R.O. : la preuve en est qu'elles ne sont presque jamais réalisées par des chercheurs opérationnels mais par des organisateurs ou par des techniciens de l'entreprise qui ont assimilé la représentation en réseau et le délicieux algorithme du plus court chemin. Cela est d'ailleurs bien normal, car dans une application P.E.R.T., la quasi-totalité du travail concerne le recueil, la critique et la diffusion de l'information.

D'autre part, si l'on examine la manière dont les entreprises effectuent leur programmation, on observe un effacement progressif des modèles au 
fur et à mesure qu'on passe du long terme au court terme. Est-ce un caractère structurel ou bien une situation passagère ?

Enfin, force est de constater que c'est, la plupart du temps, à des informaticiens ou à des organisateurs que les entreprises confient les problèmes du court terme.

Tout cela justifie, à nos yeux, que le corps des chercheurs opérationnels se pose à des questions et se demande, par exemple, si la programmation à court terme ressortit, dans la pratique, à l'Organisation et à l'Informatique, l'artillerie lourde de la Recherche Opérationnelle étant définitivement mal adaptée à cette guerre de mouvement? $\mathrm{Ou}$, d'une manière plus constructive, qu'il examine quels doivent être la place et le rôle de la R.O. dans l'élaboration des programmes de gestion à court terme.

Les réponses à de telles interrogations requièrent des confrontations d'expérience et des investigations qui trouveront leur place dans un groupe de travail spécialisé de la section Recherche Opérationnelle de l'AFCET (1).

\section{Qu'est-ce que la programmation à court terme (P.C.T.)}

Il faut bien commencer par définir ce dont nous parlons. Programmation, chacun sait ce que cela signifie : par exemple, fixer les valeurs d'un groupe de variables qui soient compatibles avec des contraintes et réalisent au mieux certains objectifs. Quant au court terme, cela est certainement moins clair car, suivant les cas, le terme d'une P.C.T. peut être quelques heures (par exemple 8 heures dans une usine travaillant à 3 équipes), la journée, la semaine, la quinzaine. Noús dirons qu'on est dans le court terme, lorsque les sorties de la P.C.T. (le programme) sont directement branchées sur l'exploitation et le contrôle d'un système.

Donnons quelques exemples.

La P.C.T. dans une usine, c'est l'ordonnancement de la production; ainsi, s'il s'agit de fabrication mécanique en petite série, l'ordonnancement fixera chaque semaine l'ordre de prise en charge des divers lots de pièces sur les diverses machines (problème du "job-shop ").

Les problèmes d'affectation de moyens matériels et humains relèvent également de la P.C.T.; ainsi une compagnie aérienne doit programmer mensuellement l'affectation du personnel de bord aux divers vols et procéder ensuite à des réajustements quotidiens.

On rencontre également tous les problèmes dits "de transport" qui se présentent sous des formes variées. Donnons l'exemple de la programmation quotidienne des transports de pondéreux dans l'agglomération moscovite. Tous les transports entre les diverses usines productrices et consommatrices et les magasins sont effectués par une entreprise nationale qui dispose de 50000 camions et de 130 garages. Chaque jour en

(1) Consulter pour une inscription à ce groupe le secrétariat et les bulletins de I'AFCET. 
fin d'après-midi, toutes les demandes de transport pour le lendemain sont transmises à un organisme planificateur central : celui-ci prend en compte ces demandes et la situation, prévue pour le soir, des camions dans les garages (état final du programme de la veille); il établit ensuite des trajets partant d'un garage, desservant un certain nombre de points et revenant à un garage et affecte un ou plusieurs trajets à chaque camion. Les ordres de route de chaque véhicule arrivent chaque jour dans les garages vers 5 heures du matin.

On pourrait citer bien d'autres problèmes; chacun de nous en a certainement plusieurs en tête.

La caractéristique commune des programmes à court terme, c'est de s'appliquer à une réalisation particulière de l'ensemble des variables qui caractérisent l'état du système à contrôler.

Que la périodicité de ces programmes soit la journée, la semaine ou la quinzaine, ce n'est pas tant cela qui marque la P.C.T., mais bien le fait qu'on ne peut pas tabler sur la loi de distribution des variables, ou utiliser des moyennes ou opérer des regroupements donnant une bonne stabilité statistique.

Première conséquence : la réalisation particulière qui constitue l'état initial $E_{1}$ du système (que le programme devra transformer en un état final $E_{2}$ conformément à certains critères) devra être saisie par une quête d'informations sur le terrain renouvelée à la période quotidienne, hebdomadaire... de la P.C.T.; d'où la nécessité d'une infrastructure organisationnelle considérable.

Deuxième conséquence : les modèles, si modèles il y a, devront posséder une variété (au sens cybernétique) considérable, c'est-à-dire une richesse et une capacité de contrôle élevées; ils seront donc complexes et chers.

\section{L'effacement apparent des modèles quand on passe du long terme au court terme}

Mais trouve-t-on souvent des modèles dans les applications concrètes de programmation à court terme ? Pour le savoir, allons rendre visite à une entreprise normalement développée en matière "d'études scientifiques" et cherchons à prendre connaissance de quelques réalisations caractéristiques.

Tout d'abord, on va nous exposer une étude à 10 ans d'un programme d'investissement : on nous montre, en fait, des rapports et peut-être des dossiers techniques où nous trouvons des modèles de prévision économique, un modèle de choix et de sélection des variantes, un modèle d'optimisation de la gestion financière; les sorties de ces modèles sont des équations, des listings, des courbes, etc. En bref, exposer une étude à long terme, c'est présenter des modèles et leur conclusions.

Supposons ensuite que nous nous intéressions à la programmation annuelle dont le but est de répartir la production d'un certain nombre de catégories de produits entre diverses usines ou équipements, de prévoir 
les besoins en hommes et les horaires, de dénouer des choix entre soustraitance et accroissement de certaines capacités de production, etc. On va peut-être nous présenter les résultats de simulations d'activité dans diverses hypothèses et nous dire "l'ordinateur a montré que....". Mais, sans gratter beaucoup, nous découvrirons bientôt des modèles (quelque peu de programmation linéaire par exemple) ne couvrant peut-être pas la totalité des questions et, sans doute, complétés par des ajustements au départ et à l'arrivée.

Si maintenant nous demandons à examiner la programmation à court terme, par exemple, l'ordonnancement de la production de l'usine A, que verrons-nous? Probablement des moyens et des procédures. Les moyens : un ordinateur, des bons de travaux (imprimés ou cartes perforées) des fichiers auprès du chef d'ateliers, etc. Les procédures : on nous expliquera que les commandes arrivent au service commercial, qu'elles ont examinées, que le premier arrivé est le premier servi, qu'elles sont codifiées puis perforées, que l'ordinateur émet des bons de travaux qui vont au bureau de planning et que celui-ci charge les machines (suit une longue explication mettant en évidence tous les cas particuliers, les récriminations du commercial, les acrobaties qu'il faut faire tous les jours, etc.).

Mais où sont les modèles ?... Peut-être n'en existe-t-il aucun d'explicite. Mais même si un modèle, par exemple de job-shop a été élaboré, il a de grandes chances d'être fortement effacé, car recouvert d'une gangue épaisse de règles et de procédures d'organisation et de traitement de l'information, gangue secrétée par le milieu pour raccorder le modèle à la réalité complexe et vivante de l'usine. Un tel effacement est-il inéluctable ? Certainement pas dans tous les cas, mais la réponse doit être modulée suivant les problèmes et le contexte des entreprises.

Il semble cependant qu'il sera toujours plus difficile de créer et de faire survivre des modèles au fur et à mesure qu'on se rapproche du court terme pour en arriver finalement au temps réel; et ceci pour des raisons techniques tout d'abord, la complexité des systèmes technologiques à contrôler et la diversité des situations instantanées; ensuite, pour des raisons de finalité.

En effet ce que cherche une entreprise qui se lance dans des études à long terme, c'est une vision synthétique et "décidable ». Les modèles étant, par définition, des structures qui apportent des réponses aux questions posées, tout effort de rationalité dans le domaine du long terme conduit donc à la recherche de modèles (opérationnels, économiques, sociologiques...).

Par contre, lorsqu'il s'agit de gestion à court terme, d'exploitation courante, de contrôle, ce que cherche l'entreprise c'est un système, asse mblage de règles, de procédures, de moyens humains et automatiques permettant de maîtriser la complexité de la vie industrielle. Mais pour concevoir un tel système, il faut connaître à fond la réalité et donc l'analyser : le métier de l'analyse (organisateur ou informaticien) c'est de casser les problèmes en fragments simples et rassurants tels que chacun d'eux puisse être contrôlé par une instruction élémentaire. 
Quand un problème et la réalité qu'il recouvre, sont ainsi cassés en menus morceaux, il est très difficile et il faut un grand effort de volonté pour remonter à un niveau synthétique qui mette en évidence des structures générales. D'autant plus que la juxtaposition des instructions élémentaires constitue en tout état de cause un programme, certes non optimal, souvent partiellement compatible, mais qui présente au moins la qualité d'être proche des phénomènes réels et donc d'être localement adapté et adaptable.

On pourrait enfin ajouter quelques considérations pratiques qui vont dans le sens de l'effacement des modèles. Ainsi, les entreprises sousorganisées, dans lesquelles l'information est défectueuse, insuffisante, biaisée, espèrent trouver dans une première mise en ordre apportée par les analyses d'organisation et d'informatique, une valeur ajoutée à la gestion ; cet espoir masque le gain supplémentaire à attendre d'une modélisation. Vont-elles réllement trouver cette valeur ajoutée? La question est d'importance et il serait intéressant d'en rechercher la réponse par des études de cas.

D'un autre côté, les entreprises fortement "informatisées » sont sous la coupe des informaticiens et il est prouvé que le chercheur opérationnel n'y est pas toujours personna grata. Autre domaine d'investigation.

\section{La place et le role de la R.o. dans la programmation à court terme}

En résumé, la programmation à court terme a pour objet de bâtir des systèmes de contrôle (de pilotage) de phénomènes complexes; pour ce faire elle part d'une analyse de détail de la réalité. La faiblesse des pratiques courantes consiste à rester au ras d'une description anecdotique sans remonter au niveau des modèles explicatifs : déviation du praticien.

A l'opposé, la programmation à long terme a pour objet d'apporter des vues de synthèse, ce qui conduit à l'élaboration de modèles. La faiblesse de certaines études "académiques " c'est de ne pas ancrer les modèles sur une analyse suffisante de la réalité : déviation du théoricien.

La vocation générale de la R.O. nous semble ressortir de ces constatations : remédier aux faiblesses des études et pallier les déviations des théoriciens et des praticiens; c'est-à-dire, d'une part ancrer les modèles théoriques dans la réalité et d'autre part montrer la voie permettant de remonter de descriptions anecdotiques vers la modélisation.

Mais, pour passer de la généralité de cette vocation à une définition plus précise du rôle et de la place de la Recherche Opérationnelle dans la programmation à court terme, il faut pousser plus loin les investigations pour pouvoir répondre à des questions telles que les suivantes :

- La programmation mathématique, sous ses diverses formes, est-elle adaptée aux exigences du contrôle à court terme de systèmes complexes ? Quelle stationnarité structurelle requiert-elle? (par exemple, le programme des transports de Moscou " bénéficie " d'un système économique rigide). 
- Quel est le gain d'efficacité à attendre du remplacement des heuristiques naïves que sont les règles de bon sens utilisées par les praticiens, par des modèles heuristiques plus largement finalisés ?

- Si le contrôle des systèmes semble échapper à une modélisation fine, du moins est-il possible de bâtir des modèles qui fourniront des règles de gestion que viendront compléter ensuite des procédures de régulation. Si done l'attitude cybernétique doit prendre le pas, comment la Recherche Opérationnelle s'insère-t-elle dans la conception des systèmes de contrôle et régulation?

- La mise en ordre de l'infrastructure d'organisation et d'informatique doit-elle nécessairement précéder la réflexion sur les modèles ? $\mathrm{Ne}$ court-on pas le risque d'en rester toujours au niveau des moyens et des procédures et de ne ja mais arriver à la remise en question des méthodes de gestion?

- Dans quel cas ce risque présente-t-il un enjeu important ? Autrement dit, quelles sont les situations ou la modélisation apporte une valeur ajoutée appréciable et celles où elle est un luxe pour gens raffinés ?

- Enfin, on pourrait se demander quelle doit être la balance, dans les problèmes du court terme entre R.O. Recherche Opérationnelle = élaboration de modèles de gestion, et $\mathrm{RO} \equiv$ Rationalité Opératoire $=$ articulation logique des analyses, des études et des réalisations pour améliorer les situations existantes ? Corrélativement, quels sont le rôle et la place de la Recherche Opérationnelle, de l'Organisation et de l'Informatique ?

Tout chercheur opérationnel qui s'est quelque peu colleté avec des applications à court terme des méthodes de R.O. a certainement des idées et des éléments de réponse aux questions précédentes; sans doute aussi, d'autres questions le tourmentent.

C'est pourquoi nous pensons qu'il est intéressant que puisse s'instaurer un large échange de vues entre experts, qui permettra sans aucun doute de dégager des conclusions utiles aux chercheurs opérationnels et aussi, bien sûr, aux utilisateurs. 